\title{
Stroke in women (Sudanese experience)
}

\section{${ }^{1}$ Shadia Ibrahim MSc, ${ }^{* 2}$ Abbashar Hussein MD PhD, ${ }^{3}$ Amira Sidig MD, ${ }^{4}$ Faroug Yasin MD, ${ }^{5}$ Ahmad Babikir Mohmad MD, ${ }^{6}$ Osman Gadour FRCP, ${ }^{7}$ Omer-Aladil MD, ${ }^{8}$ Mohamed Saad MD, ${ }^{9}$ Ahmed Hamad MD, ${ }^{10}$ Mohmad Malk Aldar MBBS and ${ }^{11}$ Esam Mahmoud Ahmad MBBS.}

\author{
${ }^{1}$ Pharmasist, Elshaab Teaching Hospital-Sudan. \\ ${ }^{* 2}$ Corresponding author, Consultant Neurologist, Associated Prof. of Medicine, University of \\ Khartoum, Faculty of Medicine, Department of Medicine, Neurology section. Sudan, \\ P.O.Box102 Khartoum, Sudan, Tel. +249-9123456722. E-mail: abbashar59@yahoo.com. \\ ${ }^{3}$ Consultant Community Physician, Associated Prof., University of Elnilin-Sudan. \\ ${ }^{4}$ Consultant Neurologist, Prof. of Medicine, Omdurman Islamic University-Sudan. \\ ${ }^{5}$ Consultant Physician, Associated Prof. of Medicine, University of Elnilin-Sudan \\ ${ }^{6}$ Consultant Physician, Prof. of Medicine, Omdurman Islamic University-Sudan. \\ ${ }^{7}$ Physician, Associated Prof. of Medicine, University of International Africa-Sudan \\ ${ }^{8}$ Consultant Neurosurgeon, University of Elnilin-Sudan. \\ ${ }^{9}$ Physician, Associated Prof. of Medicine, University of Bahr Elgazal-Sudan. \\ ${ }^{10}$ Senior Registrar of Medicine, University of Eltigana. Sudan. \\ ${ }^{11}$ Registrar of Medicine. Federal Ministry of Health. Sudan.
}

\begin{abstract}
Cerebrovascular Accident (CVAs) represents a major cause of death and disability among women. Age, hypertension, diabetes mellitus, hyperlpidemia, obesity and heart disease are known predisposing factors for the development of stroke. To describe clinical presentation of (CVAs) and to identify risk factors among adult Sudanese female.: This prospective cross sectional hospital based study it was done in (ETH) ELshaab Teaching hospital, in the period between April 2007 and July 2008. 309 Sudanese female where included in the study. The common age group affected was between $70-79$ years (27.2\%), followed by age group 60-69 (21.4\%).Limbs weakness, symptoms in favour of cranial nerves involvement, headache, convulsion, loss of conscious, were the main presenting symptoms. Hypertension is the most common risk factor. Considerable number of our patients had protein $\mathrm{C}$, protein $\mathrm{S}$ and Antithrombin 111 deficiency, rare risk factors included anti phospholipids syndrome. The clinical presentation of CVA among Sudanese female dose not differed from what was reported worldwide except there is increased incidence of protein $C$, protein $S$ and Antithrombin 111 deficiency among our studied group.
\end{abstract}

Keywords: Sudanese, women, stroke

\section{INTRODUCTION:}

Stroke is a major cause of death, it is third only to coronary artery diseases and cancer, and ranks the second most frequent cause of neurological morbidity in the developed world. It accounts for 150, 000 to 175,000 deaths per annum. Women and men share many of the same risk factors for stroke like age , family history .diabetes, , hypertension, smoking ,coronary artery disease, a trial fibrillation ,lack of exercise, obesity ,excessive alcohol use ,previous stroke and history of transient ischemic attack. ${ }^{1}$ There are several risk factors that may be more prevalent in women than in men. African- American and Alaska native women are particularly likely to have high blood pressure. High level of LDL cholesterol also raises the risk of stroke in both men and women, however, cholesterol levels in women tend to remain lower than men until after menopause, when women's cholesterol may increase significantly, this places post menopausal women at a higher risk of stroke. ${ }^{2}$ The low-dose oral contraceptives currently prescribed for birth control carry an increased risk of 
stroke. The risk is more pronounced among smokers, women with history of migraines and women over the age of 40 . Women's risk of stroke increase during pregnancy end in the weeks just after delivery. During this time, women are particularly at risk for a hemorrhagic stroke. Women who have had frequent miscarriages suffer from anti phospholipids syndrome which is a disorder that causes excessive blood coagulation and it also increases the risk of stroke. Women who experience migraines have a 3 to 6 fold increase in stroke risk. And migraines occur more frequently in women than in men. Women are also vulnerable to type of stroke known as stroke secondary to fibro muscular dysphasia [FMD] which is an inherited condition in which the patient experiences ongoing destruction of arterial blood vessels, including those in the neck that supply blood to the brain and arteries in the brain it self .Stroke related to FMD primarily affects women especially those over the age of 50). Stroke is defined as a sudden onset of focal or generalized neurological deficit due to vascular lesion. The symptoms of a stroke are related to the anatomical location of the damage .Types of stroke: 1) Complete Stroke: This term implies a neurological deficit which has reached its maximum extent, usually within less than six hours limit of the onset.2) Stroke in evolution: Describes evolving and deteriorating symptoms and signs, usually more than six hours from the onset.3) Transient ischemic attack (TIA): This denotes a focal deficit, such as a weak limb, aphasia or loss of vision, lasting from a few second to 24 hours followed by complete recovery. ${ }^{3}$

To study the patterns of clinical presentation of stroke and to identify risk factors among adults Sudanese female seen in Al-Shaab Teaching Hospital, from January 2006 to January 2008.

\section{MATERIALS AND METHODS}

This is a descriptive prospective, hospital based, cross sectional study. Sudan is the largest country in Africa, covering one eighth of the continent surface with an area of 2.5 million sq $/ \mathrm{kms}$. More than 2000 $\mathrm{km}$ from north to south, having diverse environment due to different climatic zones, extending from the great desert to equatorial rainy forests. Since its independence in 1956, Sudan has witnessed only eleven years of peace. The civil wars, inter-ethnic conflicts, floods, droughts and variant patterns of rain, have had adverse effects on the economic and the developmental status of the whole country. Sudan shares extensive borders with nine countries. The population of Sudan according to 2007 census was 38 millions. The annual growth rate also increased from 1.9 percent to 2.6 percent.

The study was conducted at Al Shaab Teaching Hospital which is a tertiary hospital, located in the centre of Khartoum state. There are two neurological units with 43 beds and two neurosurgical units with 50 beds, there are three intensive care units, two neurology referred clinic and three neurosurgery referred clinics each week. The study population included 309 female patients with stroke, referred or admitted to Al Shaab Hospital in the period from January 2006 to January 2007. All the patients were newly diagnosed and they were not on treatment. All the patients were adult Sudanese female, those below 18 years of age were excluded. All patients gave their verbal consent to participate in the study. Ethical approval was received from the local ethics committee prior to the start of the study .The data was collected by the authors using structural questionnaire. The informers were either the patients or close relatives. A full details history was taken from each patient, the history included, Age, Sex, origin and the presenting neurological symptoms (upper and lower limbs weakness, headache, convulsion, loss of consciousness, cranial nerve symptoms, unsteadiness, speech disturbances sphincter defects. Also past medical history (similar illness, loss of vision, head trauma HTN and DM), drugs history eg contraceptive pills, family history (similar illness, neurological illness during childhood) and social history (alcohol consumption or smoking). After finishing the history, proper systemic and neurologic examination was performed to each patient by the others including cardiovascular, respiratory system ,abdomen, musculoskeletal, high cerebral function, cranial nerves including fundal examination, cerebellar function, motor and sensory systems. The physical signs were grouped into neurological signs and systemic signs. Investigations done for each patient include CXR, ECG, Echocardiography, CBC \& ESR, RBS, serum cholesterol and triglyceride, $C T$ scan of the brain, while the investigations done if needed included ( T3, TSH, T4, protein C, protein S and Antithrombin 111,Anticardiolibin antiphospholipid Antibodies, level VDRL, HIV screen, LP and CSF examination). The data were introduced in the computer from a master sheet recording using software program, the data were analyzed and the results were expressed into figures, tables or graphs using statistical package for social science (SPSS) and discussions, conclusions and recommendations were done. 
Am. J. Sci. Ind. Res., 2011, 2(2): 157-160

\section{RESULTS}

Age distribution range between 19-95 years. 6 patients their age was 19 years $(1.9 \%), 39$ between $20-29$ years (12.6\%), 18 between $30-39$ years $(5.8 \%), 21$ between $40-49$ years $(6.8 \%), 51$ between $50-59$ years (16.5\%) , 66 between 60-69 years $(21.4 \%), 84$ between $70-79$ years ( $27.2 \%)$ and 24 patients their aged a above 80 years $(7.8 \%)$. Almost 123 one patients (40\%) were from Khartoum State, 60(19\%) from Gazera State, 42 (13.8\%) from the North, $36(11.7 \%)$ from East, 36 (11\%) from the West and only 12 patients (3.6\%) were from the South. Risk factors: 129 patients (51.5\%) had hypertension, 87 (28.2\%) had DM, 33 (9.7\%) had hyperlipidemia, 27 (8.7\%) had ischemic heart disease, $27(8.7 \%)$ had protein $\mathrm{C}$, protein $\mathrm{S}$ and Antithrombin 111 deficiency, 18 (5.8\%) had TIA, 15 $(4.9 \%)$ tend to smoke cigarettes, $15(4.9 \%)$ had recurrent stroke, 12 (3.9\%) had CHF, 12 (3.9\%) had past history of DVT, 12 patients(3.9\%) used to take contraceptive pills, obesity was observed in 12 patients (2.9\%),3 (2.9\%) had AF,9 (2.9\%) had hypothyroidism, $9(2.9 \%)$ had past history of recurrent attack of abortion, and three patients had anti phospholipids syndrome . Onset: 213 patients had sudden onset of the disease (68.9\%), while 96 patients presented with gradual onset (31.1\%).

Clinical presentations: Weakness was the presenting symptom in 300 patients $(99,1 \%)$ The right side was affected in (58.3\%) while the left side was affected in (41.7\%),symptoms in favour of cranial nerves involvement in 177 patients( 57.3\%), headache in 114 patients (36, 9\%), convulsion in 63 patients $(20,4 \%)$, loss of conscious in 18 patients (5,8\%), neck stiffness in 12 patients (3.4\%) while sphincteric disturbances was noticed in 9 patients (2.3\%). Out of 309 patients 180 had facial cranial nerve palsy while 15 patients (4.9\%) had an evidence of second cranial nerves involvement and only nine patients $(2.9 \%)$ showed an evidence of involvement of other cranial nerves.

CT brain findings: 186 patients had ischemic infarction (59.2\%), 96 patients had hemorrhage (29.1\%), 9 patients $(2.7 \%)$ had hemorrhagic infarction, 12 patients had normal CT brain (3.4\%), and four patients had subdural hematoma (1.9\%). It did appear that 183 patients improved (58.3\%), 117 were static (35.8\%) and nine died (2.9\%).

\section{DISCUSSION}

Stroke is an important cause of death and disability, however no systematic study has been conducted to elucidate possible causes of stroke among Sudanese patients. The mean age of our patients was (56.61 years) this is less than what was reported in the industrialized countries, for instance in the Framingham study the mean age of stroke patients was (66.1 years) for women and (65.4 years) for men. Most properly this due to the fact that patients with risk factors (like hypertension and DM) in the developing countries like Sudan are facing the problems of lacking the facilities to follow them regularly so they are more liable to develop the complications associated with these diseases e.g. stroke.The high percentage of stroke among patients from Khartoum State, where the study was conducted and Gazera State, and low percentage in the East, West and North, may be explained by the awareness of population and availability of diagnostic tools and health facilities to which the patients used to attend in Khartoum and Gazera State, while illiteracy, poverty and distant health facilities or lack of diagnostic tools explain the low presence in the Western, Eastern and Southern regions. Classical risk factors for development of CVAs are more prevalent among older women. Similar to what was reported by other researchers the most frequent risk factor among our patients was hypertension (51.5\%), followed by diabetes mellitus (28.2\%),hyperlipidemia and smoking. ${ }^{4}$ Other risk factors were ischemic heart disease, protein $\mathrm{C}$, protein $\mathrm{S}$ and Antithrombin 111 deficiency, recurrent stroke, CHF, past history of DVT, contraceptive pills, obesity, AF, hypothyroidism and anti-phospholipids syndrome. Striking the natural anticoagulants (protein $\mathrm{C}$, protein $\mathrm{S}$ and antithrombin 111) were at the lower range of normal in most of our patients. In women taking contraceptive pills, the coagulation derangement caused by the pills probably increased the thrombotic potential of the low normal levels of the natural anti-coagulant proteins. ${ }^{5-}$ ${ }^{6}$ Out of 309 patients with stroke only one had anti phospholipids syndrome and she had frequent miscarriages. Women's risk of stroke increase during pregnancy and just after delivery. During this time, women are particularly at risk for a hemorrhagic stroke. ${ }^{7-8}$ One of our patients presented with venous infarction as a complication of sagital sinus thrombosis following delivery and this a well known complication which tend to occur during pregnancy. Psychological disturbances should also be considered especially among female who came from 
the west (36 patients), who faced the social and economical out put of the prolonged war. Neurological symptoms and signs were found to be similar to what was mentioned in the literature, weakness in the upper and lower limps was found to be the commonest neurological symptoms among our studied group, followed by headache, convulsion and loss of consciousness. ${ }^{9-10}$ Ischemic infarction was found to be the commons cerebral insult in stroke patients (54.4\%), this in keeping with the international figures of prevalence of cerebral infarction and cerebral hemorrhage. ${ }^{11-12}$ With regard to outcome $(38.8 \%)$ of the patients were improved, $(28.2 \%)$ were static and three of the total died $(2.9 \%)$ the mortality rate was in the lower range, similar to vilalta and arboix's Barcelona registry. ${ }^{13}$

\section{CONCLUSION:}

1) The prevalence of incidence of stroke in female increases with increased age.

2) The major risk factors for ischemic and hemorrhagic stroke were hypertension, Diabetes and hyperlipidemia respectively.

3) Limbs weaknesses, headache convulsion, loss of consciousness were the main presenting symptoms.

4) Cerebral or ischemic infection had a higher frequency than cerebral hemorrhage.

\section{RECOMMENDATIONS:}

1) Health education considering life style, diet, exercises, regular checkup of blood pressure glucose etc., should be considered to minimize the incidence of stroke.

2) Further studies are needed to verify our suggested explanation and to find out why women seem to have a lower quality of life after a stroke than men.

\section{REFERENCES:}

1. Koh, KK; Mincemoyer, R; Bui, MN; Csako, G; Pucino, F. Effects of hormone-replacement therapy on fibrinolysis in postmenopausal women. $\mathrm{N}$ Engl J Me 1997;336:683-690.

2. Cote, R; Wolfson, C; Solymoss, S; Mackey, A; Leclerc, JR. Hemostatic markers in patients at risk of cerebral ischemia. Stroke 2000; 31:1856-1862.

3. Smith, A; Patterson, C; Yarnell, J; Rumley, A; BenShlomo, Y. Which hemostatic markers add to the predictive value of conventional risk factors for coronary heart disease and ischemic stroke? The Caerphilly study. Circulation 2005; 112:3080-3087.
4. Coull BM. Anticoagulants and antiplatelet agents in acute ischemic stroke. Report of the Joint Stroke Guideline Development Committee of the American Academy of Neurology and the American Stroke Association (a division of the American Heart Association). Stroke 2002; 33(7): 1934-1942.

5. Latchaw REI. Guidelines and recommendations for perfusion imaging in cerebral ischemia. Stroke 2003;34(4): 1084-1104.

6. Sacco RL. Guidelines for prevention of stroke in patients with ischemic stroke or transient ischemic attack. Stroke 2006; 37(2): 577-617.

7. Straus SE. New evidence for stroke prevention: Scientific review. JAMA 2002; 288(11): 1388-1395.

8. Fung TTI. Prospective study of major dietary patterns and stroke risk in women. Stroke 2004; 35: 2014 2019.

9. Wassertheil-Smoller S.. Effect of estrogen plus progestin on stroke in postmenopausal women. The Women's Health Initiative: A randomized trial. JAMA 2003; 289(20): 2673-2684.

10. Adams RJ. Coronary risk evaluation in patients with transient ischemic attack and ischemic stroke: A scientific statement for healthcare professionals from the Stroke Council and the Council on Clinical Cardiology of the American Heart Association/American Stroke Association. Circulation 2003; 108(10): 1278-1290.

11. Strans SE, Majumdar SR, Mc aliter FA."New evidence for stroke prevention: scientific review"JAMA 2002; 288 (11):1388-95.

12. Liu S. Whole-grain consumption and risk of ischemic stroke in women. JAMA 2000; 284(12): 1534-1540.

13. Vilalta JL. Arboix A. The Barcelona Stroke Registry. Eur Neurol 1999; 41: 135-142. 